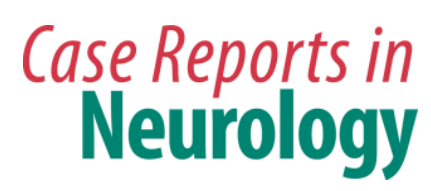

\title{
Endoscopic Surgery for Traumatic Acute Subdural Hematoma
}

\author{
Hiroyuki Kon Atsushi Saito Hiroki Uchida Mizuho Inoue \\ Tatsuya Sasaki Michiharu Nishijima \\ Department of Neurosurgery, Aomori Prefectural Central Hospital, Aomori, Japan
}

\section{Key Words}

Endoscopic surgery · Trauma · Acute subdural hematoma

\begin{abstract}
Traumatic acute subdural hematoma (ASDH) is generally addressed by craniotomy under general anesthesia. We report a patient whose traumatic ASDH was treated under local anesthesia by one-burr-hole endoscopic surgery. This 87 -year-old woman had undergone coil embolization for a ruptured right middle-cerebral artery aneurysm and placement of a ventriculoperitoneal shunt for normal pressure hydrocephalus 5 years earlier. Upon admission, she manifested consciousness disturbance after suffering head trauma and right hemiplegia. Her Glasgow Coma Scale score was 8 (E2V2M4). Computed tomography (CT) demonstrated a thick, left-frontotemporal ASDH. Due to her advanced age and poor condition, we performed endoscopic surgery rather than craniotomy to evacuate the ASDH. Under local anesthesia, we made a burr hole in her left forehead and increased its size to 15 $\mathrm{mm}$ in diameter. After introducing a transparent sheath into the hematoma cavity with a rigid endoscope, the clot was evacuated with a suction tube. The arterial bleeding point was electrically coagulated. A postoperative CT scan confirmed the reduction of the hematoma. There was neither brain compression nor brain swelling. Her consciousness disturbance and right hemiplegia improved immediately. Endoscopic surgery may represent a viable method to address traumatic intracranial hematomas in some patients.
\end{abstract}

(C) 2014 S. Karger AG, Basel

\section{Introduction}

Due to its minimal invasiveness, some institutions now choose endoscopy rather than craniotomy to evacuate hypertensive intracerebral hematomas. However, there are no reports on the use of endoscopic surgery to address traumatic intracranial hematomas, in

Hiroyuki Kon, MD, PhD

Department of Neurosurgery

Aomori Prefectural Central Hospital

2-1-1 Higashi-Tsukurimichi, Aomori 0308553 (Japan)

E-Mail hkon@sc4.so-net.ne.jp 
particular, acute subdural hematomas (ASDH). We report a patient with traumatic ASDH who underwent successful endoscopic hematoma evacuation.

\section{Case Report}

An 87-year-old woman who had undergone coil embolization for a ruptured middlecerebral artery aneurysm and placement of a ventriculoperitoneal shunt for normal pressure hydrocephalus 5 years earlier fell and hit her head. She suffered consciousness disturbance and was transported to our hospital $6 \mathrm{~h}$ after the fall. At the time of admission, she was confused and manifested right hemiplegia. Her Glasgow Coma Scale score was 8 (E2V2M4). A computed tomography (CT) scan showed a thick, (2 $\mathrm{cm}$ in diameter) leftfrontotemporal ASDH and a marked midline shift (fig. 1). Her pupils were isocoric and her light reflex was prompt.

The possibility of impending brain herniation required emergency evacuation of the ASDH. As her advanced age and poor condition rendered general anesthesia risky, we opted for endoscopic evacuation under local anesthesia. In case this proved impossible, we simultaneously prepared for performing a craniotomy under general anesthesia. With her head in a neutral position, we made a single burr hole at a site on her left forehead. We then increased the hole to $15 \mathrm{~mm}$ in diameter in order to obtain a wide-angle endoscopic field. As the skin incision was along a wrinkle, it raised minimal cosmetic concerns. We introduced a rigid endoscope with a tip angle of $0^{\circ}$ (Machida Endoscope Co. Ltd., Tokyo, Japan). After incising the dura, we observed a dark red clot. There was no outer membrane associated with chronic subdural hematoma. After evacuating the clot beneath the burr hole, we used the rigid endoscope to introduce a transparent sheath $(10 \mathrm{~mm}$ in diameter) into the hematoma cavity. Although a portion of the soft clot was hard, it could be removed easily with a suction tube (fig. 2a). Irrigation with artificial cerebrospinal fluid (Artcereb ${ }^{\circledR}$, Otsuka Factory, Tokushima, Japan) yielded a clear operative field and precise manipulation was possible. Upon removing the hematoma, we noted arterial bleeding on the brain surface. We applied a suction tube to the bleeding point (fig. 2 b, c) and coagulated it electrically (fig. 2 d). After confirming the removal of the hematoma and complete hemostasis, we filled the subdural space with artificial cerebrospinal fluid. The burr hole was covered with a ceramic burr-hole button and the skin incision was sutured cosmetically. The entire procedure was completed in $47 \mathrm{~min}$.

A postoperative CT scan showed an obvious reduction of the hematoma; there was no brain shift or swelling (fig. 3). Her consciousness disturbance and right hemiplegia improved immediately. She was discharged 30 days later without any neurological deficits.

\section{Discussion}

The prognosis of ASDH tends to be poor due to brain edema and/or herniation [1], so urgent surgical intervention is essential. Craniotomy under general anesthesia is usually required to evacuate the hematoma and to coagulate arterial bleeding. However, in patients who are in a poor condition and/or of advanced age, this invasive procedure can be risky. Mahoney et al. [2] reported the usefulness of burr-hole trepanation. While burr-hole surgery is performed in the emergency room to treat patients with a CT diagnosis of brain hematoma [3], additional craniotomy may be necessary because the rate of hematoma evacuation via a single burr hole can be low. On the other hand, advances in endoscopic instrumentation may facilitate the evacuation of intracerebral hematomas $[4,5]$. 
There are few reports on endoscopic treatment of traumatic intracranial hematomas. Shiomi and Shigemori [6] considered endoscopic surgery for chronic subdural hematomas to be advantageous and they presented an endoscopically guided method in which a drainage tube is inserted into the hematoma cavity. Kessel et al. [7] used spinal endoscopy to treat acute, traumatic, anterior epidural hematomas of the cervical spine. However, endoscopic surgery has not been used for the removal of ASDH because it is difficult to observe the entire hematoma through the burr hole and hemostatic procedures cannot be implemented. A flexible endoscope must be used to facilitate approaching the hematoma from all sides if the trepanation is located at the center of thick hematomas. Furthermore, limited suction capacity renders hematoma evacuation through the working channel of flexible endoscopes impossible.

In our patient, we were able to remove the hematoma through a single burr hole on the forehead because we could set a long trajectory along the hematoma (fig. 4). In addition, we could place a strong suction tube in the port sheath and we were able to obtain a clear operative field by irrigating the hematoma cavity. This made it possible to coagulate the bleeding point.

While we were able to take advantage of the forehead curvature of the skull to introduce the transparent sheath into the hematoma, this approach route is not available in all patients. Due to our patient's advanced age and poor condition, we chose endoscopic evacuation rather than craniotomy; we suggest that this technique be considered to treat ASDH in such patients to avoid the risks posed by general anesthesia. Some ASDH patients may indeed require craniotomy to obtain external decompression to avoid severe cerebral edema or a significant increase in intracranial pressure [8], so endoscopic surgery, which is simple and less invasive than craniotomy, can only be considered in a limited number of cases. This technique should be applied for elderly patients who have brain atrophy and a relatively thick hematoma because the endoscopic manipulation favors a wider subdural space. On the contrary, craniotomy entailing external decompression should be considered for cases associated with the rapid aggravation of disturbed consciousness or brain contusion. Motohashi et al. [9] treated 2 patients with ASDH on the posterior cranial fossa by single burr-hole evacuation in the emergency room. Although our patient underwent endoscopic surgery in the operating room, it is also possible to use this procedure in the emergency room.

More cases must be collected in order to document the utility and safety of endoscopic ASDH evacuation and to further improve the technical aspects of this procedure. Endoscopic surgery for ASDH evacuation holds promise because this procedure is less invasive than craniotomy, saves time and facilitates the rapid reduction of increased intracranial pressure.

\section{Disclosure Statement}

The authors report no conflict of interest concerning this report.

\section{References}

1 Haselsberger K, Pucher R, Auer LM: Prognosis after acute subdural or epidural haemorrhage. Acta Neurochir (Wien) 1998;90:111-116.

-2 Mahoney BD, Rockswold GL, Ruiz E, Clinton JE: Emergency twist drill trephination. Neurosurgery 1981;8:551-554.

-3 Springer MF, Baker FJ: Cranial burr hole decompression in the emergency department. Am J Emerg Med 2004;6:640-646. 
-4 Auer LM, Deinsberger W, Niederkorn K, Gell G, Kleinert R, Schneider G, Holzer P, Bone G, Mokry M, Korner E, Kleinnert G, Hanusch S: Endoscopic surgery versus medical treatment for spontaneous intracerebral hematoma: a randomized study. J Neurosurg 1989;70:530-535.

-5 Cho DY, Chen CC, Chang CS, Lee WY, Tso M: Endoscopic surgery for spontaneous basal ganglia hemorrhage: comparing endoscopic surgery, stereotactic aspiration, and craniotomy in noncomatose patients. Surg Neurol 2006;65:547-555.

6 Shiomi N, Shigemori M: The use of endoscopic surgery for chronic subdural hematoma (in Japanese). No Shinkei Geka 2005;33:785-788.

7 Kessel G, Bocher-Schwarz HG, Ringel K, Perneczky A: The role of endoscopy in the treatment of acute traumatic anterior epidural hematoma of the cervical spine: case report. Neurosurgery 1997;41:688-690.

-8 Jiang JY, Xu W, Li WP, Xu WH, Zhang J, Bao YH, Ying YH, Luo QZ: Efficacy of standard trauma craniectomy for refractory intracranial hypertension with severe traumatic brain injury: a multicenter, prospective, randomized controlled study. J Neurotrauma 2005;22:623-628.

-9 Motohashi O, Kameyama M, Shimosegawa Y, Fujimori K, Sugai K, Onuma T: Single burr hole evacuation for traumatic acute subdural hematoma of the posterior fossa in the emergency room. J Neurotrauma 2002;19:993-998.

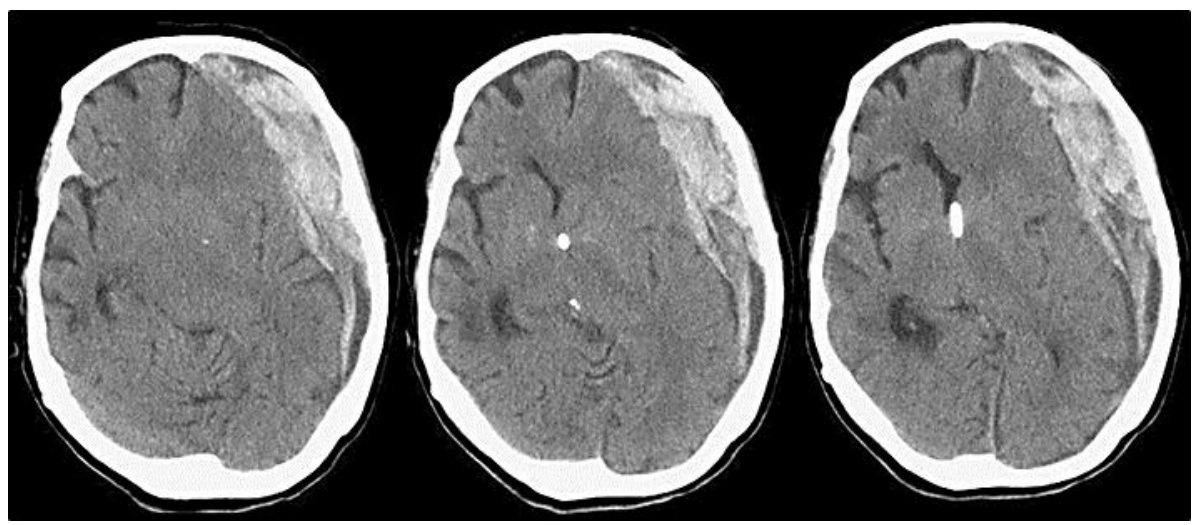

Fig. 1. CT scans obtained at admission demonstrate a thick, left-frontotemporal ASDH (2 cm in diameter) and a marked midline shift. 
Kon et al.: Endoscopic Surgery for Traumatic Acute Subdural Hematoma

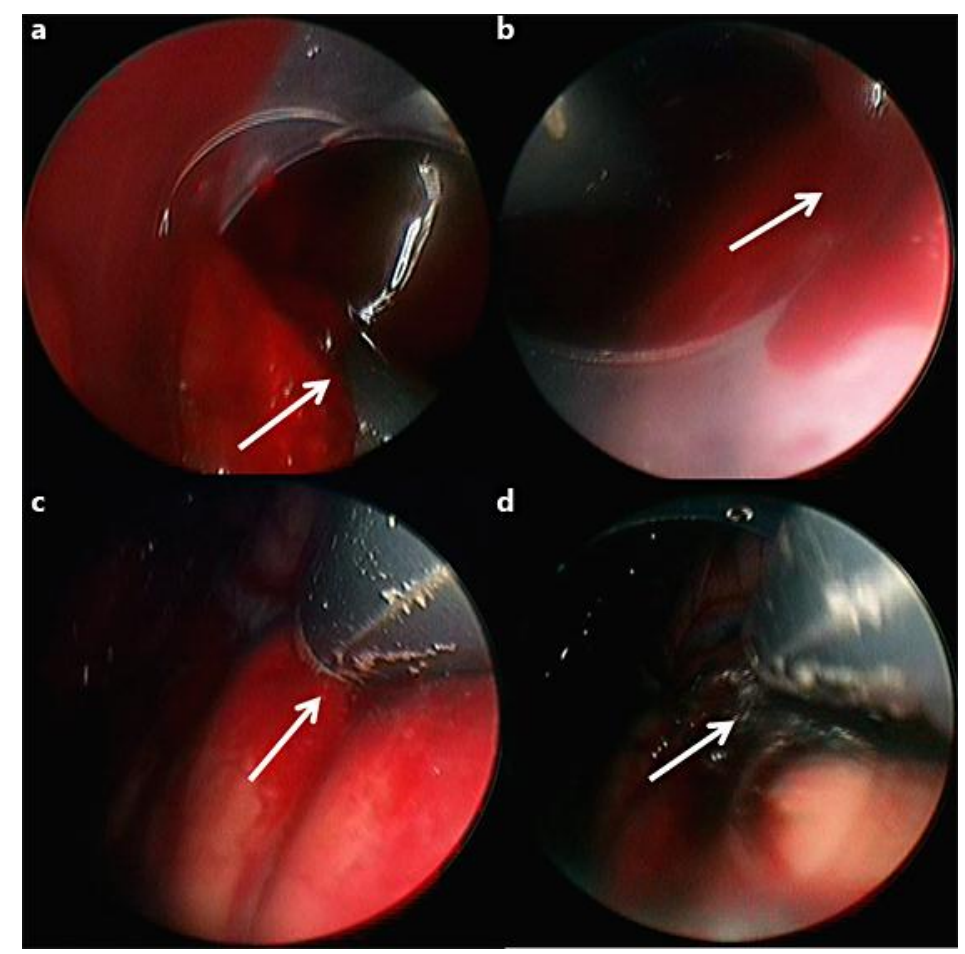

Fig. 2. Intraoperative images. a ASDH is aspirated with a suction tube (arrow). b Bleeding from a thin artery on the brain surface (arrow). c Arterial bleeding is controlled with a suction tube (arrow). $\mathbf{d}$ Electric coagulation of the bleeding point (arrow).

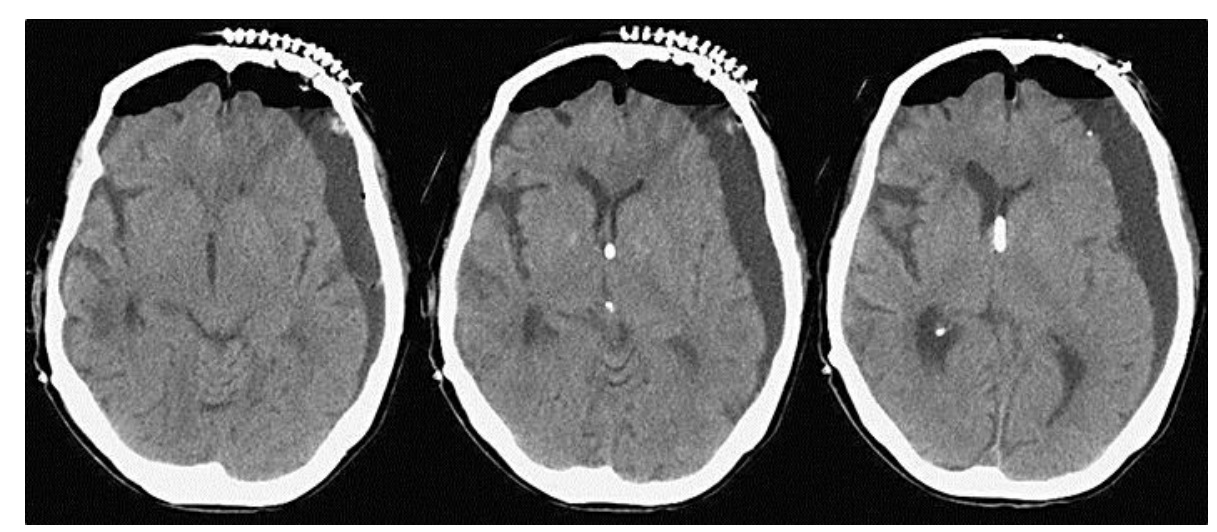

Fig. 3. Postoperative CT scans demonstrating the reduction of the hematoma and improvement in the midline shift. 

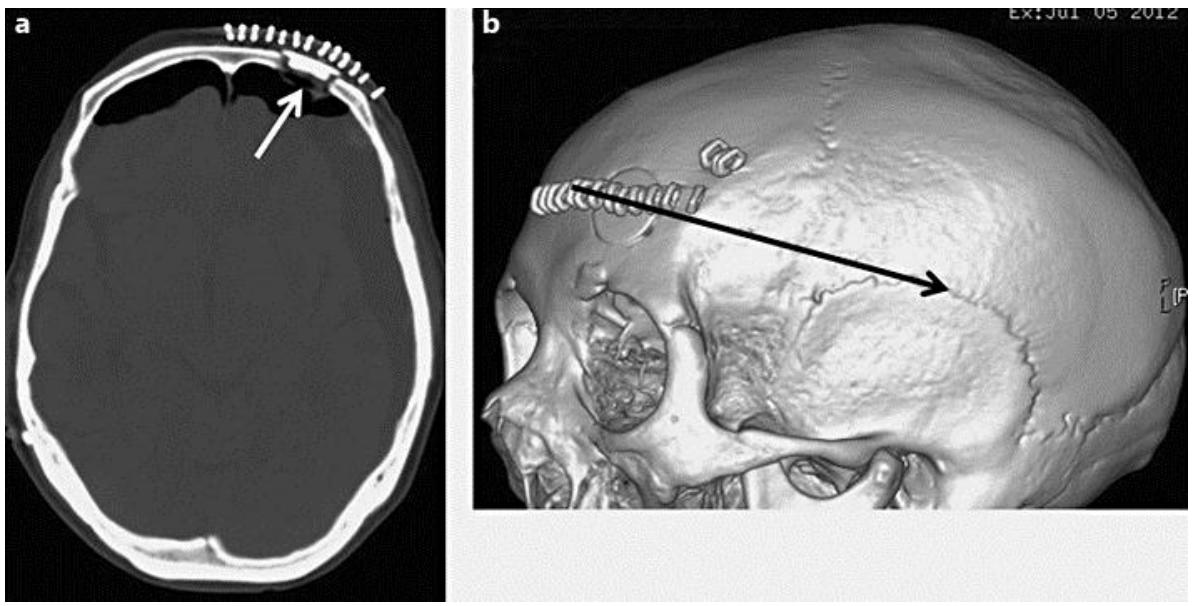

Fig. 4. Postoperative CT scans. a A bone image showing the burr-hole button (arrow). b 3-Dimensional surface image showing the operative trajectory along the ASDH (arrow). 